\title{
El proceso de enseñanza-aprendizaje en grupos heterogéneos de la Universidad Metropolitana del Ecuador
}

\author{
Dr. Raúl Rodríguez Muñoz ${ }^{1}$, Universidad Metropolitana del Ecuador, Ecuador \\ Recibido: $\quad 8$ de octubre, 2017. \\ Aceptado: $\quad 26$ de junio, 2018.
}

\section{Resumen}

En la Universidad Metropolitana del Ecuador, la organización del proceso de enseñanza y aprendizaje en la asignatura "Fundamentos del marketing", incluye estudiantes de diferentes carreras. Esta situación resulta un tanto compleja para los docentes de ese curso, pues al tener un grupo tan diverso, se da una brecha en los aprendizajes logrados por los estudiantes. Esta composición, además, coincide con otros estudios de referencia en la denominación de heterogéneos, ya que cuentan con estudiantes de varias carreras y edades distintas. Por eso, el objetivo del presente artículo es proponer un modelo de organización del aprendizaje para dicha asignatura, utilizando el método sistémico estructural y la modelación. La propuesta se denomina "Modelo del grupo de aprendizaje" y se fundamenta en las estrategias didácticas y experiencias del aprendizaje cooperativo. Considera además, los contenidos y el diagnóstico inicial para organizar en subgrupos a los estudiantes con diferentes carreras, y permite que el docente guíe el proceso hasta el producto integrador, mediante deberes. Como resultado, los estudiantes desarrollan los temas en conjunto durante el periodo 39, realizan las tareas con un enfoque multi-carrera y transmiten saberes y experiencias propias, hasta la presentación del producto integrador. En conclusión, el "Modelo del grupo de aprendizaje" deriva en acciones de organización, lo que permite lograr mejores resultados de aprendizaje y desarrollo de habilidades en los estudiantes.

Se corroboran las ideas de Hernández-Selles, González-Sanmamedy y MuñozCarril (2013), Madariaga, Correa, Oria y Walliser (2015) y García y Mena (2016) referentes al aprendizaje cooperativo y colaborativo. Asimismo, se emplea una planificación coherente con las actividades docentes.

\section{Abstract}

\section{The teaching-learning process in heterogeneous groups from} the Metropolitan University of Ecuador

The structure of the teaching-learning process in the Fundamentals of Marketing subject includes students from different careers, a complex situation for the tea-

1 El profesor Raúl Rodríguez es Doctor en Ciencias Pedagógicas, Máster en Educación y Profesor Titular de la Universidad Metropolitana del Ecuador. Actualmente se desempeña como Coordinador de Investigaciones de la Universidad Metropolitana del Ecuador. Contactos: rrodrguez1@gmail.com y rrodriguez@umet.edu.ec.
Raúl Rodríguez Muñoz. El proceso de enseñanza-aprendizaje en grupos heterogéneos de la Universidad Metropolitana del Ecuador. Revista Comunicación. Año 39, volumen 27, número 2, julio-diciembre, 2018. Instituto Tecnológico de Costa Rica. ISSN: 0379-3974 / e-ISSN1659-3820. 
cher, since its heteronomous nature implies they learn and develop skills with a different mindset, creating a gap in the learning achieved by the students. This group composition fits that of other reference studies where heterogeneous students are defined as multiracial and of different ages. Therefore, the objective of this article is to propose a learning organization model for the Fundamentals of Marketing subject. A "learning group model" is proposed utilizing the systemic structural method and modeling, based on cooperative learning didactic strategies and experiences. The contents and the initial diagnosis in the model are taken into account to organize students with different careers into subgroups, with the teacher guiding the process by assigning tasks to reach an integrating product. As a result, the students develop 39 the topics as a whole, carry out tasks with a multi-career focus, and transmit knowledge and their own experiences leading up to the presentation of the integrating product. In conclusion, the "learning group model" derives in organizational actions, which fosters better learning results and skill development in the students. The ideas of Hernández (2013), Madariaga (2015) and García (2016) regarding cooperative and collaborative learning, and use of coherent teaching activity planning are corroborated.

\section{INTRODUCCIÓN}

En la Universidad Metropolitana del Ecuador (UMET) los grupos de estudiantes para las asignaturas del ciclo básico se organizan bajo una composición de diferentes carreras, como "Gestión Empresarial", "Diseño Gráfico", "Contabilidad y Auditoría", "Gestión de Empresas Turísticas y Hoteleras", "Licenciatura en educación inicial", "Desarrollo del talento infantil", "Optometría", "Sistemas de la Información" y "Derecho", entre otras.

Para nadie es un secreto que lograr el aprendizaje en una asignatura como "Fundamentos del marketing", la cual no es necesaria para el posterior desempeño de muchos de los estudiantes de carreras ajenas a la disciplina, es un reto tanto para el estudiante como para el docente, ya que son de especialidades distintas y las experiencias varían de grupo en grupo y por periodos o semestres.

La asignatura "Fundamentos del marketing" pretende aproximar al estudiante en la comprensión del proceso mediante el cual las personas -así como también los grupos- obtienen aquello que necesitan y quieren, creando productos con valor e intercambiándolos en el mercado. El estudiante se familiariza con las bases del marketing desde los aspectos generales que involucra la dirección de la empresa, a fin de que entienda y conozca el mercado para relacionarlos con los hábitos, al igual que las costumbres de los consumidores, de acuerdo con la necesidad cambiante de la sociedad de consumo.

Se configura el espacio para que desarrolle habilidades para trabajar sobre su preparación futura en el manejo de competidores que impulsen a las empresas a indagar sobre nuevas oportunidades, nue- vos mercados, así como posibles nuevos productos. La asignatura permite iniciar a los estudiantes en el conocimiento de las herramientas necesarias para la investigación, la elección de los mercados, el desarrollo, la definición de productos y los servicios.

Además, también es de hacer notar que los grupos heterogéneos, como el que se forma con esta asignatura, incluyen una mezcla que también puede abarcar desde etnias, géneros, culturas y edades diferentes, hasta experiencias de vida y opiniones. Esta particularidad se ajusta a las condiciones de trabajo en grupos que reciben otras asignaturas en esa casa de estudios, como "Realidad Socio Económica y Cultural" y "Matemática básica".

Así las cosas, se formuló entonces la siguiente pregunta de investigación: ¿Cómo reducir la brecha de aprendizaje en el curso "Fundamentos de markenting", entre los estudiantes de la carrera "Gestión Empresarial" y los de las restantes disciplinas? La problemática se sustenta en la supuesta ventaja de los primeros al contar con aprendizajes básicos de la especialidad y haber matriculado una asignatura propia para ellos. Además, se eligió como objetivo el siguiente: Modelar una organización del aprendizaje en la asignatura "Fundamentos del marketing", con la cual se pueda mejorar la adquisición de conocimientos significativos por parte de los discentes.

\section{MATERIAL Y MÉTODOS}

En este apartado se explica cómo se hizo la propuesta de modelo de grupo de aprendizaje y la metodología de la investigación, así como la forma en la que se abordó como material, los procesos y productos investigados y la manera en la que se aplicó cada método. 


\subsection{Participantes}

Al inicio del período 39, impartido desde abril hasta agosto de 2017, se decidió hacer una propuesta de organización para dos grupos, con la asignatura de "Fundamentos del marketing". La muestra se compuso por esos dos grupos de estudiantes. El primero, proveniente de las carreras de "Contabilidad y Auditoría", "Gestión de empresas turísticas y hoteleras", Gestión empresarial", "Licenciatura en educación inicial", y "Desarrollo del talento infantil", y el segundo grupo elegido correspondía a las áreas de "Sistemas de la Información" y "Diseño Gráfico".

El primer grupo, se caracterizó por diferencias de edades entre 30 y 50 años, con intereses distintos socialmente y fuentes de empleo también dispares.

El diagnóstico inicial consistió en la aplicación de un cuestionario de tres temas para ambos grupos. El primer tema planteó qué es el marketing, el segundo, su importancia para su profesión y el tercero, las soluciones que ofrece esta disciplina. Se hallaron diferencias entre los que cursan la carrera de "Gestión Empresarial" y los restantes de otras carreras, ya que para ellos es fundamental la asignatura y ya tienen identificados los contenidos y habilidades necesarias, por lo que les resulta más fácil comprender los temas.

De igual modo, fueron definidos dos marcos muestreales: el grupo B y el B-1. Cada uno constituye un caso para comparar los resultados. La definición de estos se basó en sus características esenciales ya señaladas: rango de edad y oficios.

\subsection{Instrumentos}

El conocimiento del nivel empírico se estudió mediante instrumentos. Para ello, se definió una metodología mixta con elementos del estudio de caso:

a. El método analítico-sintético.

b. La observación para profundizar primero en la esencia de la supuesta brecha de aprendizaje y proporcionar un nuevo enfoque al docente para dirigir el proceso de enseñanza-aprendizaje de la asignatura, y así, usar la comparación para definir entre un grupo y otro. c. La discusión grupal en las actividades del curso.

Para elaborar la propuesta, se emplearon el método sistémico-estructural y la modelación. Finalmente, para la observación se utilizaron guías de observación. Además, la discusión grupal contó con la definición de uno o varios objetivos que guiaron la aplicación en los sujetos seleccionados, luego de las observaciones.

Es importante hacer notar que la modelación (como método) se caracteriza por propiciar la mediación entre la realidad y el objeto de investigación, en representación teórica o práctica, pero de forma sintética. Entonces, como la organización del proceso de enseñanza-aprendizaje del curso "Fundamentos del marketing" es compleja y en la universidad se asume desde diferentes formas, fue posible probar de manera experimental la propuesta de modelo, así como encontrar un referente y aplicar su estructura mediante acciones.

El instrumento de diagnóstico que se aplicó a los estudiantes fue validado por docentes considerados especialistas, y el proceso fue realizado en dos oportunidades, con un mes de diferencia. De la primera sesión resultó que se debían establecer mejoras para las preguntas 2 y 3 , y ya con la segunda, todos quedaron satisfechos con la propuesta.

\subsection{Procedimiento}

Para el desarrollo del proceso investigativo se partió del análisis del objeto (organización del proceso de enseñanza-aprendizaje). Con la aplicación del método analítico-sintético se llegó entonces a la definición de los criterios e indicadores de diagnóstico, para comparar los resultados en los dos grupos.

Por su parte, la definición de ideas que sustentaron la organización del proceso de enseñanza-aprendizaje, partió de estudios de mayor proximidad ${ }^{2}$ a la definición de los grupos heterogéneos y su reconocimiento en las universidades.

2 Existen formas generales de organización del proceso en los niveles educativos que tienen que ver con la finalidad y los objetivos de las actividades docentes. En cada una de ellas se manifiesta una manera interna en que se organiza y participa el estudiante. Si son de diferentes carreras, asumirán el conocimiento y la formación de la habilidad de manera distinta. Es común que -incluso al sentarse- los estudiantes se agrupen por sus carreras si se trata de una conferencia o clase práctica. 
Es importante hacer notar que las universidades ajustan el proceso docente con base en estas formas de organización. Entonces, es tarea para el docente de las asignaturas afines definir y encontrar estrategias específicas para guiar el proceso de enseñanzaaprendizaje ante grupos con múltiples carreras que se identifican como heterogéneos.

El método analítico-sintético se evidenció en la valoración y definición de los referentes teóricos que se emplearon y sustentaron este trabajo. Se destacan aquellos que emitieron ideas que fundamentaron esta investigación.

Para Feito (2015) existen ventajas y desventajas ante estos fenómenos, sean intencionales o dadas las circunstancias de la organización. Como una desventaja, señala la diferencia de edades, lo cual lleva al docente a subestimar a los estudiantes de menor edad dentro del grupo. No obstante, Feito (2015) profundiza en las ventajas, entre ellas la identificación de redes naturales de apoyo para el aprendizaje, dejando atrás erróneas concepciones del trabajo grupal. Finalmente, señala que el papel del maestro es similar al director de teatro, que tiene que guiar un gran concierto.

En otra perspectiva, Peirats y López (2013) hacen referencia a estrategias didácticas para trabajar con la diversidad en grupos de estudiantes. En este sentido, concuerdan con la opinión de trabajar en grupos interactivos, lo cual -apoyado en la guía de un supervisor voluntario- supone mejorías en el aprendizaje. Por su parte, Fernández de Haro (2014) se refiere al aprendizaje cooperativo o en comunidades de aprendizaje, y puntualiza que en el sistema cooperativo cada alumno alcanza la meta que se ha propuesto en la medida en que los compañeros de su grupo consiguen las suyas; además resalta la importancia de asumir las ventajas del aprendizaje cooperativo o sea, incluye el esfuerzo propio, pero también el colectivo, donde el estudiante ve a su compañero no como un rival, sino como un colaborador.

De tal caso, es evidente que la problemática se manifiesta en diferentes contextos y ha sido presentada de distinta manera. Sin embargo, el aprendizaje cooperativo se plantea como una forma de presen- tar soluciones y mejorar los resultados de la formación de las habilidades.

Por otra parte, no es posible olvidar el vínculo de la universidad con la sociedad. Analizar qué ocurre y qué se espera de esta institución tiene en consideración sus procesos gobernantes, estratégicos y sustantivos. Por eso, al analizar el objetivo 5 del Plan Nacional "Toda una Vida" significación de vital importancia para la asignatura, ya que plantea la transformación de la matriz productiva, integrando los productos, los procesos productivos y las relaciones sociales resultantes de esos procesos (SENPLADES, 2017).

Más adelante se hace énfasis en las micro, pequeñas y medianas empresas, las cuales deben tratar de alinearse con esas actividades de formación e incorporar valor agregado a sus actividades, con el fin de fortalecer sus capacidades. Toda la sociedad y sus profesiones contribuyen con esta meta, de acuerdo con su nivel, por lo que no puede ser desconocido, sino identificado como un objetivo de la formación de los profesionales de cualquier carrera. En este caso, la asignatura le permite al profesional enfrentarse a los desafíos del entorno y tomar en cuenta dichas actividades para sus decisiones. Su objetivo general ("Describir las variables de la mezcla de marketing a través de la revisión de los principios del producto, precio, distribución y comunicación, para ser aplicados en las organizaciones con o sin fines de lucro donde se desempeñen laboralmente" (programa de asignatura P-39, p. 2) así lo plantea.

En este sentido, un estudiante de cualquier carrera debe saber que en el marketing son aprendizajes necesarios su definición, las terminologías relacionadas (mercado, consumidor final), así como también los niveles de la planeación organizacional en cuanto al contenido de un plan anual de marketing y el monitoreo ambiental. Otro elemento de importancia son los factores macro-ambientales externos

3 El "Plan Nacional de Desarrollo 2017-2021 Toda una Vida" de Ecuador es el principal instrumento del Sistema Nacional Descentralizado de Planificación Participativa (SNDPP), y su objetivo es contribuir al cumplimiento progresivo de los derechos constitucionales, los objetivos del régimen de desarrollo y disposiciones del régimen de desarrollo (a través de la implementación de políticas públicas) y los programas, proyectos e intervenciones que de allí se desprenden. Más información, en el sitio https:// observatorioplanificacion.cepal.org/es/planes/plan-nacional-dedesarrollo-2017-2021-toda-una-vida-de-ecuador. 
y los micro-ambientales externos, así como relacionarse con la comprensión del ambiente interno, mercado de consumo y comportamiento de compra de los consumidores.

Igualmente, son requisitos importantes tener preparación con respecto a la toma de decisiones del consumidor (el proceso de decisión de compra), comprender la jerarquía de necesidades de Maslow, y demostrar su conocimiento en la investigación de mercados, su importancia y las técnicas de investigación. No menos fundamental resulta que el estudiante muestre conocimiento sobre la segmentación de mercado, el mercado de consumidores y el mercado empresarial. Como habilidades se describen las siguientes:

- Exponer claramente la importancia del marketing en la empresa.

- Identificar las oportunidades y amenazas en función del análisis externo.

- Identificar el proceso de toma de decisiones del consumidor.

- Describir el mercado y las variables de segmentación del mercado de consumidores finales.

El estudiante debe obtener un desempeño, así como los productos que evidencian el logro en la asignatura, aspecto común y aplicable a otras materias. En particular, para el estudio realizado si se tiene en consideración estos resultados y se valora su composición, se puede proponer un diagnóstico de entrada que permita apreciar con qué conocimiento llegan los estudiantes. Es un elemento metodológico de importancia para organizar el proceso de enseñanza-aprendizaje.

Se considera que en el nivel universitario se llega con percepciones y determinados grados de conocimiento acerca de un tema específico. Lejos queda el criterio de ver al estudiante como un sujeto vacío. En opinión de quien escribe, el estudiante siempre posee una apreciación y un conocimiento acerca del tema. Por eso es importante saber qué medida o nivel posee al respecto. No obstante, en el caso de la Universidad Metropolitana del Ecuador, el es- tudiante de tercer semestre puede matricular esta asignatura y tener más conocimiento que otros, lo que se aprecia con más fuerza en los que provienen de la carrera "Gestión Empresarial".

Entonces, conseguir que el docente haga un buen diagnóstico es fundamental, ya que se asume como punto de partida para dirigir el proceso de enseñanza-aprendizaje y elaborar la guía para que el estudiante logre estrategias de aprendizaje. Incluso pueden estar visualizadas en el aprendizaje cooperativo, el colaborativo o en el trabajo en grupo. En todo caso, se platean cuáles fueron los instrumentos aplicados para el referido diagnóstico y qué aspectos se evaluaron.

La configuración del instrumento de diagnóstico partió del propósito general de la asignatura y luego tuvo en cuenta los objetivos específicos para definir los contenidos. Los indicadores para el diagnóstico se orientaron desde el análisis de los aprendizajes, su relación con los contenidos y habilidades a demostrar, lo que se sintetiza en las evidencias de aprendizaje, tal y como se muestra en la tabla 1.

Con la aplicación del método analítico-sintético se llega entonces a la definición de los siguientes criterios e indicadores de diagnóstico:

1. Importancia de los procesos de marketing para la profesión del estudiante:

a) Logra hacer corresponder la importancia de los procesos de marketing con su profesión.

b) Establece la relación moderada de la importancia de los procesos de marketing con su profesión.

c) El nivel de relación de la importancia de los procesos de marketing con su profesión es bajo.

2. Identificación de los procesos de marketing y conceptos

a) Marca los procesos de marketing.

b) Solo marca tres de los procesos de marketing.

c) No marca ninguno de los procesos de marketing. 
El proceso de enseñanza-aprendizaje en grupos heterogéneos de la Universidad Metropolitana del Ecuador

Tabla 1

Evidencias de aprendizaje del programa de la asignatura Fundamentos del Marketing $P$-39

\section{Resultados de aprendizaje}

El estudiante explicará la importancia del marketing en la actividad empresarial.

El estudiante estará en la capacidad de diseñar estrategias de marca, empaque y etiqueta para un producto.

\section{Evidencias}

\section{Desempeño}

Producto
Elabora un mapa conceptual sobre Mapa conceptual.

la importancia del marketing en actividad empresarial.

Crea nombres de marca para pro- Nombres de marca para productos ductos.

Identifica estrategias de empaque y Realización de estudios de casos. etiqueta para un producto.

El estudiante estará en la capaci- Diseña canales de distribución Ejercicios en los que el estudiante dad de diseñar canales de distribu- para diferentes casos de estudio. diseña canales de distribución para ción para la empresa. empresas en función del tipo de producto. Estudios de casos.

El estudiante estará en la capaci- Realiza talleres en clase en los que Ejercicios en los que aplica estratedad de describir la importancia del precio con relación a los otros aplica ciertas estrategias de precio. gias de precios. Estudios de casos.

elementos de la mezcla de marketing.

El estudiante estará en la capacidad de identificar variables de comunicación que puede utilizar para la empresa.
Lleva a cabo talleres en grupo en Ejercicios donde aplica estrategias los que aplica estrategias comuni- comunicacionales para empresas, cacionales. productos o servicios.

Estudios de casos.

Tomado y adaptado del programa de la asignatura Fundamentos del Marketing P-39 adaptados por el docente

\subsection{Estrategia de análisis}

El instrumento de diagnóstico se orienta a relacionar al estudiante con la problemática del proceso de enseñanza-aprendizaje de la asignatura "Fundamentos del marketing" y, además, informa acerca de su nivel de conocimiento y habilidades de entrada con respecto a los procesos de marketing.

De igual modo, la observación participante del profesor (cuya intención era apreciar las reacciones de los estudiantes ante la aplicación del instrumento de diagnóstico) permitió contar con otro nivel de información de enfoque psicológico o psicosocial. No se trató de una observación común, sino de una observación científica que estructuró desde un objetivo: determinar el comportamiento de los estudiantes respecto a los contenidos del marketing.
Además, se utilizó un registro de observación compuesto por tres columnas, en el cual se reflejaron los nombres y apellidos de cada estudiante. Cada columna reflejó de manera individualizada las reacciones. No fue complejo hacer este instrumento, ya que se contó con el listado oficial digitalizado y se elaboró la tabla con las características descritas.

Para el final de ese primer encuentro, se programó una discusión grupal, último método empleado para lograr la información pertinente. En este sentido, el instrumento consistió de cuatro preguntas, las cuales facilitaron los datos respectivos. Las opiniones de los estudiantes sobre los procesos de marketing y cómo mejorar la enseñanza-aprendizaje fue el objetivo, teniendo en consideración que existen estudiantes de diferentes carreras y que, además, es una expectativa saber para qué les sirve profesionalmente la asignatura. 
Por su lado, la estructura de la discusión grupal tuvo las siguientes interrogantes:

a. ¿Cómo considera que debe ser el desarrollo de la asignatura, práctica, teórica o con una combinación de ambos enfoques?

b. ¿Cuáles son sus criterios respecto a los procesos de marketing? ¿Qué conoce de ello?

c. ¿Puede definir qué aportes puede hacer a su profesión el conocimiento de los procesos de marketing?

d. ¿Cuáles son las expectativas que posee y cómo se ve usted al concluir el periodo 39?

Luego de la aplicación de estos métodos y técnicas aquí relacionadas, al concluir la primera actividad de clases de dos horas de tiempo asignado, el docente consideró oportuno hacer una triangulación de los resultados obtenidos de cada método o técnica, orientado a ganar más rigor en el estudio. Obsérvese que cada instrumento guarda una relación con sus ítems, lo que posibilita valorar su comportamiento y futuros resultados. Contrastar los métodos y técnicas antes de su aplicación asegura que exista una relación coherente y esa es la razón por la cual la metodología seguida es aplicable para otros periodos.

\subsection{El modelo de grupo de aprendizaje}

Como puede apreciarse hasta aquí, con la aplicación de los métodos que fueron descritos y sus resultados, se está en condiciones de configurar una propuesta que se denomina modelo, ya que como representación ideal de las relaciones organizacionales sirve como guía teórica para desarrollar las acciones que se deriven de este en el proceso de enseñanza-aprendizaje de la asignatura "Fundamentos del marketing".

Finalmente, se considera oportuno apoyarse en la modelación y el método sistémico-estructural para concebir el modelo que facilite el desarrollo del proceso de enseñanza-aprendizaje y de las habilidades que se pretende lograr en los estudiantes.

En la aplicación tradicional de los sistemas de aprendizaje para grupos heterogéneos se dividiría el grupo en subgrupos según carrera o se buscaría afinidad entre los estudiantes. Se agruparía teniendo en cuenta quizás sus capacidades y se les entregarían las tareas a realizar en la clase. Sin embargo, el problema aquí radica en que cada carrera debe aprovechar los conocimientos adquiridos para usarlos como básicos en su gestión y de acuerdo con el diagnóstico, existen desniveles en la percepción y grados diferentes de conocimiento respecto a los procesos de marketing. Estos son retos por resolver para aprovechar la diversidad y no ver en esto sus debilidades.

Por otra parte, con base en el aprendizaje cooperativo y desde el método sistémico-estructural, se estableció una nueva estructura de aprendizaje y desarrollo de habilidades, parte de principios lógicos, tal y como sigue:

1. Combinar el trabajo individual y cooperativo.

2. Distinguir el trabajo de equipo como esencial.

3. Incrementar la responsabilidad de la ayuda mutua en los estudiantes.

4. Entender que el éxito de uno del grupo es el éxito de todos y todos son responsables.

5. Compartir el currículum con los estudiantes, en una buena relación docente-estudiante.

El modelo de grupo de aprendizaje es un todo metodológico. Gráficamente se representa la forma de ordenar el conocimiento y las habilidades en función de las carreras, lo cual queda estructurado en anillos de conocimiento. Cada anillo se relaciona y cada grupo coopera con los restantes, de modo que se genera una ola de aprendizaje que bate hacia todos, hacia lo interno. Los líderes del conocimiento pueden ser de una carrera u otra; todo está en el nivel de conocimiento y percepción que tengan para aprender y aplicar soluciones.

En la figura 1, se muestra la representación, atendiendo al diseño seguido para dar respuesta a las insuficiencias de aprendizaje del grupo. En este caso, el grupo B se caracteriza por un rango de edades entre 30 y 50 años, y los estudiantes de "Gestión empresarial" fueron distribuidos en los restantes subgrupos, pues en el diagnóstico ellos demostra- 
ron tener niveles mínimos de comprensión de los problemas en procesos de marketing:

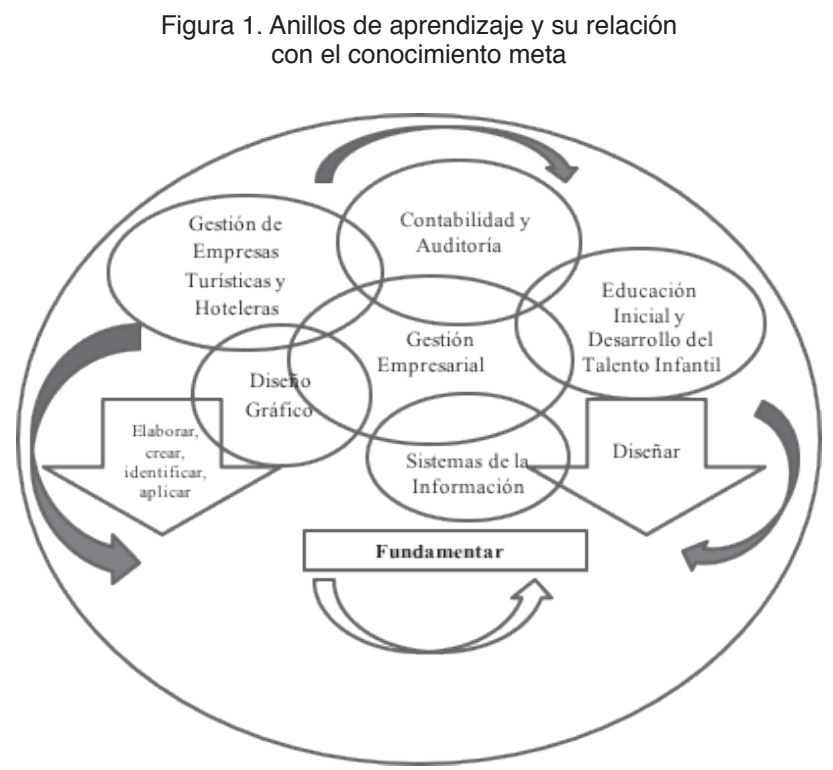

Mapa conceptual sobre la importancia del marketing en la actividad empresarial. Nombres de marca para productos. Estrategias de empaque y etiqueta para un producto. Canales de distribución. Estrategias de precio. Estrategias comunicacionales.

Como puede apreciarse, las habilidades de elaborar, crear, identificar y aplicar los conocimientos que se describen debajo de diferente color configuran la habilidad de diseñar, la cual constituye el centro durante el trabajo de los círculos del conocimiento. De este modo, desarrollar las anteriores debe conducir hacia diseñar; al menos es el propósito. Nótese que se llaman círculos del conocimiento a los que cada estudiante representa desde una carrera en el grupo y que -además- es capaz de estructurar. La habilidad fundamentar procesos también es seguida de cerca y ha sido considerada clave por los docentes, ya que el estudiante debe sustentar sus propuestas durante los ejercicios o clases prácticas. De esta manera, el conocimiento meta que se trabaje podrá ser considerado en cada actividad. Los estudiantes se identifican con su grupo y generan ideas que desde la perspectiva de su carrera les favorece para enriquecer sus habilidades en la asignatura.

Es importante destacar que es un proceso complejo, el cual se materializa mediante las formas de organización seguida por el docente. Por lo tanto, en una conferencia el funcionamiento del grupo estará dirigido a ordenar las actividades a seguir durante y después de la conferencia. En esa etapa será más efectiva, y se sugiere apoyar el aprendizaje con el uso de tecnologías y códigos actuales, como el uso de WhatsApp para comunicarse entre ellos. En otras actividades, como seminarios, talleres y clases prácticas, el trabajo de los grupos varía en su praxis, pero sigue siendo el eje para el desarrollo del proceso de enseñanza-aprendizaje y las habilidades.

\section{RESULTADOS DE LA APLICACIÓN}

El modelo constituye el referente que guía en la organización del proceso de enseñanza-aprendizaje. Para su aplicación, se concibieron acciones que connotan su aplicación, las cuales son:

a. Definición de los objetivos del grupo de aprendizaje según la composición de las carreras y definición del líder del grupo de aprendizaje.

b. Definición de las tareas del grupo, por integrantes.

c. Evaluación de sus integrantes, de forma colectiva e individual.

Una vez aplicado dicho modelo en cada actividad docente, se logró entender cuáles conceptos o contenidos se pueden reforzar más en el estudiante.

Posteriormente, se analizaron los resultados obtenidos, una vez utilizado el modelo de grupo de aprendizaje.

Para el análisis de los resultados se tuvo en cuenta que se hicieron evaluaciones sistemáticas y dos evaluaciones parciales a lo largo de todo el periodo 39 (el cual consta de 16 semanas), y que al final, los estudiantes entregaron los productos integradores, los cuales se asumieron en concordancia con otras asignaturas. Sin embargo, "Fundamentos del marketing", en este caso del grupo B, fue una asignatura asumida como el eje para este producto, y se concertó con el curso de "Metodología de la investigación". En este sentido, se consideró también si la modelación del grupo y sus efectos fueron o no positivos. 
Una vez concluido el periodo y finalizadas las valoraciones del modelo, los resultados de la asignatura son consistentes para estimar dos criterios generales de credibilidad y aplicabilidad, además de considerar la confirmabilidad.

\subsection{Comparación de los resultados}

El estudio inicial y la aplicación del diagnóstico facilitaron el inicio y la comprensión de la problemática de los grupos heterogéneos, para configurar posteriormente la propuesta de mejoramiento del trabajo del docente. Por consiguiente, bajo estos criterios generales se asumieron los resultados del diagnóstico inicial para el grupo B. El punto de partida fueron los resultados de aprendizaje de los estudiantes, cómo llegaron en la primera fase del periodo, qué necesidades de aprendizaje tenían y cómo evolucionaron una que vez se organizó el proceso atendiendo a sus características. Es importante destacar que se aplicaron los mismos instrumentos del diagnóstico inicial, lo cual facilita la comprensión de los resultados.

Los resultados del periodo en la asignatura se pueden apreciar en la figura 2:

Figura 2

Comparación de resultados, aprobados, credibilidad, aplicabilidad en el grupo B

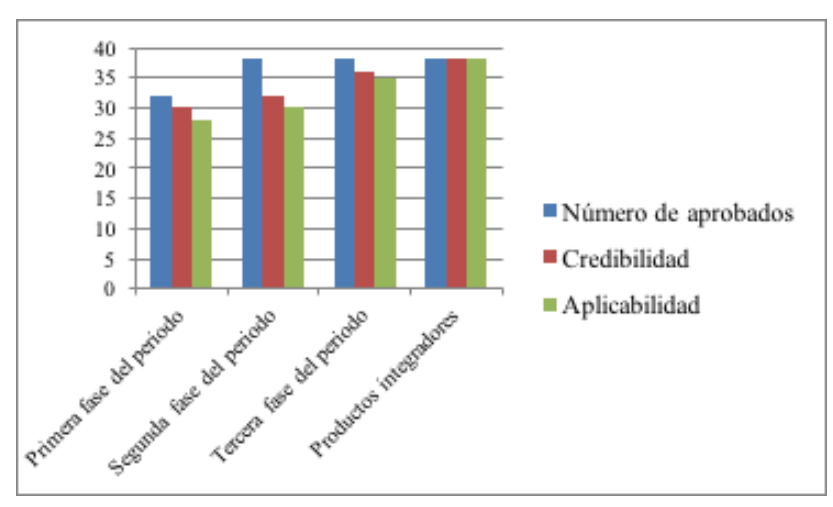

Fuente: elaboración propia

Nótese el incremento en los resultados durante la segunda etapa del periodo, que evidencia la eficiencia del trabajo con los grupos bajo el modelo descrito.

Por su parte, la credibilidad se sustenta en los resultados de la triangulación de los resultados de la observación, la encuesta y la discusión grupal, ya que estas fueron empleadas al principio del proceso de enseñanza-aprendizaje y revelaron los criterios de los estudiantes respecto a la asignatura.

De acuerdo con lo anterior, se valoran los resultados de la observación participante y se aprecia que, de un total de 38 estudiantes del grupo, solo 8 mostraron un interés por delimitar la importancia de los procesos del marketing para su profesión. Por su lado, en los niveles de búsqueda de la información de la asignatura para realizar los deberes del primer encuentro en la fase I, también se apreció la conformación de sus cuadernos, la definición y la disposición para integrar los grupos de aprendizaje. Los 8 estudiantes que actuaron diferente fueron los de la carrera de "Gestión empresarial", y los resultados de las encuestas confirman lo anterior. Solamente 8 alumnos plantearon que los procesos de marketing son importantes para su profesión.

En cuanto a conocer si aseguran de manera adecuada la atención en su centro laboral al cliente, usuario, mercado de consumidores, hubo disparidad, pues estos resultados demostraron que 26 estudiantes coincidieron con la opción de cliente, cuatro con la de usuario y 8 con la del mercado de consumidores.

De modo similar, respecto a los niveles de satisfacción en bienes o servicios que logra el centro laboral, treinta plantearon que los consideran altos, 4 que medios y 4 también los perciben como bajos.

En cuanto a responder si en el centro laboral se tienen en cuenta los deseos y necesidades de clientes la respuesta que evalúa a los usuarios tuvo 12 aprobaciones y tres al mercado de consumidores. La segunda pregunta, acerca de las definiciones y conceptos, apropiadas y correspondientes con su profesión, se obtuvieron los siguientes resultados: un total de 20 marcaron la opción orientada al proceso social y administrativo, mediante el cual los individuos y los grupos obtienen lo que necesitan y desean, creando e intercambiando valor con otros. Otros 14 se identificaron con el proceso por el cual las empresas generan valor para los clientes y establecen relaciones sólidas con ellos, por lo que obtienen a cambio valor de los consumidores. Mientras 
tanto, solo cuatro identificaron al marketing como una herramienta que se encarga de los clientes, con la cual se busca una relación perdurable con ellos y al mismo tiempo atraer más clientes.

Otro punto muy complejo de los resultados iniciales, se mostró con respecto a la oferta del marketing, y si resulta cierta o no como combinación de productos, servicios, información o experiencia que se ofrece a un mercado para satisfacer una necesidad o deseo. La segunda parte de la afirmación fue acerca de que el marketing consiste en acciones que se realizan para diseñar y mantener relaciones de intercambio deseables con audiencias meta, las cuales implican bienes, servicios, ideas u otros objetos. Aquí, igualmente los resultados se presentaron en extremo, pues 26 consideraron afirmativas o positivas las afirmaciones, cuatro más o menos y ocho que no era así.

En la discusión grupal se confirmó que hay similares resultados a lo logrado en la aplicación del cuestionario y un poco que se diferencia de los resultados de la observación. Es posible que existiera la diferencia, dado que la observación se realizó desde la misma puesta en aplicación del cuestionario.

Los resultados anteriores fueron triangulados y evaluaron la credibilidad. Para ello, se totalizaron los resultados y se sumaron para cada periodo. En la figura 2 colocada antes, se contrastan con la fase I y se pueden comparar con los resultados en las siguientes fases.

Al diferenciar los resultados obtenidos en cada método o técnica con las fuentes, se consiguieron algunas valoraciones. En primer lugar, se concluye que en cada fase los resultados variaron y mejoraron el nivel de credibilidad. La contrastación es posible si se tienen en cuenta la aplicación de métodos o instrumentos seleccionados en cada fase del periodo; así es más seguro el resultado. La credibilidad además, para los participantes, se valora porque los datos fueron compartidos con los estudiantes y estos reafirmaron que eran aceptables, es decir, creíbles.

Al consultar a otros docentes del área, la opinión fue favorable, al menos de los seis que integran el departamento de Gestión Empresarial y han trabajado con la asignatura. Algunos refirieron incluso que emplean el trabajo grupal similar al que se organizó, pero sin considerar los círculos del conocimiento. En el caso que ocupa a esta investigación, nótese que existe una tendencia que se hace corresponder con los resultados de aprendizaje del periodo respecto a un mayor número de aprobados.

De acuerdo con la lógica seguida, se consideran creíbles por el docente los resultados obtenidos. Con la operacionalización del modelo, los círculos de conocimiento y su interacción, se intensifica el trabajo cooperativo y en grupo, como bases para el desarrollo de las habilidades de la asignatura y la obtención del conocimiento.

Para valorar la aplicabilidad que se presenta también en la figura 2 (arriba), se partió de transferir el modelo a otro grupo de trabajo. Pero, en principio, se valoró la aplicabilidad con ellos mismos, o sea, se compararon los resultados dentro del grupo B. En los grupos de aprendizaje creados bajo el modelo, se sustentó la idea, ya que existen niveles diferentes y carreras distintas.

Los resultados evidenciaron que el modelo de trabajo seguido fue asumido y les aportó ventajas para el aprendizaje, se mostraron más seguros y evitó el aislamiento de estudiantes en desventajas de aprendizaje. Si se comparan los resultados ya en los productos integradores, se evidencia que los resultados fueron satisfactorios. Siguiendo la escala, todos aprobaron y los productos reunieron la calidad requerida. Además, fueron resultados reales.

Surgió, por ejemplo, Eco Jeans, una pequeña empresa estudiantil que se propone reciclar los para los pantalones de mezclilla ya usados, y ofrecer el servicio de ajustes y arreglos moderados a la prenda de vestir según solicitud del usuario. Se explicó que el grupo B poseía características en las cuales existía un rango de edades diferentes, carreras distintas y niveles variados de conocimiento inicial respecto a los procesos de marketing.

La misma metodología se aplicó en otro grupo que se denominó B-1, ya que recibía la misma asignatura. Sin embargo, se trataba de un grupo en el cual el rango de edades era aún más dispar con respecto al anterior. Se desplazaba desde los 18 hasta los 30 años. Algunos incluso, tenían un trabajo en oficios 
que no se correspondían con sus carreras, lo cual hizo más difícil el trabajo del docente, porque buscar que apliquen o desarrollen habilidades desde sus empleos no era afín. En la figura 3 se muestran los resultados comparativos de la aplicabilidad entre los grupos B y B-1:

Figura 3

Comparaciones de los resultados del grupo B y B-1 respecto a los aprobados y la aplicabilidad

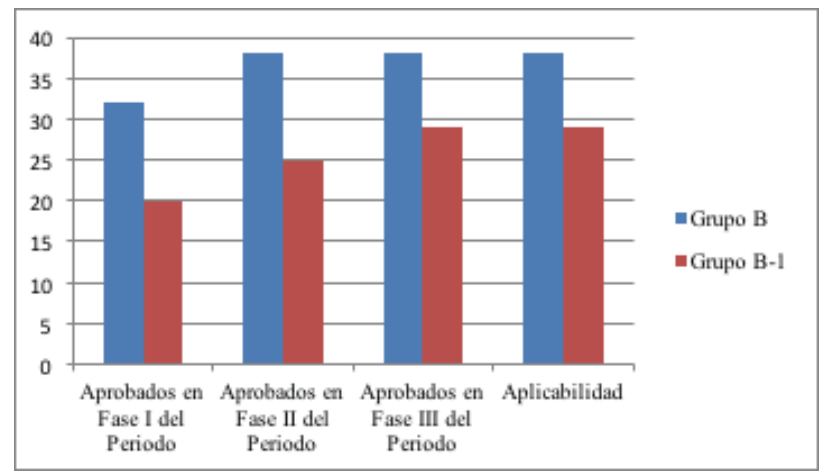

Cuando se visualizaron los datos en la figura 3, se consideró que es posible mejorar los resultados en grupos heterogéneos en relación con los resultados totales del proceso de enseñanza-aprendizaje y el desarrollo de habilidades mediante la nueva forma de organizar el proceso, ya que estos grupos son representativos de una diversidad. Se reafirma entonces la idea con los resultados de la discusión grupal.

Con respecto a la pregunta de si les era más fácil aprender o les beneficiaba su aprendizaje con esta organización, el 100\% respondió que sí, lo cual coincidió con sus expresiones durante las actividades desarrolladas, y con el total de los estudiantes de ambos grupos.

La confirmabilidad parte de lo que plantean Feito (2015), Peirats (2013) y Fernández (2014), en relación con el trabajo con grupos heterogéneos. La forma en que se pudo organizar el proceso de enseñanza-aprendizaje en la asignatura "Fundamentos del marketing" puede ser trasladada y ajustada a otros contextos concretos, grupos de estudiantes y asignaturas básicas en las cuales se ajusten grupos heterogéneos de diferentes carreras. En tal sentido, la negociación sistemática con los estudiantes genera fiabilidad y deja todo clarificado para cada caso.

\section{DISCUSIÓN Y CONCLUSIONES}

Las principales tendencias actuales incluyen el trabajo cooperativo y colaborativo en función del aprendizaje y sin duda, en el caso que ocupa este trabajo se puede afirmar que es fundamental. Aquí se coincide con John Dewey (1859-1952), precursor de este enfoque en la educación, pues en cualquier fase metodológica lo necesario es lograr la identificación de algún problema o dificultad por el estudiante y apoyado en esa experiencia avanzar en el aprendizaje. Asimismo, siguiendo a Bondi (2015) se encontró que las vías de comunicación son importantes y las relaciones entre los estudiantes de diferentes carreras pueden favorecer el aprendizaje y el desarrollo de habilidades si se enfocan a resolver problemas reales de su entorno.

En primer lugar, presentar la propuesta ante los estudiantes permitió afinar las acciones de organización grupal en la asignatura y el modelo. Además, los criterios de otros docentes reafirmaron la necesidad de mejorar la organización grupal y sacar mayor provecho para el aprendizaje de los estudiantes.

Se explica entonces qué incidencia tuvo en los estudiantes. En el grupo B se evidenció que los estudiantes diseñaron canales de distribución para empresas en función del tipo de producto. Una de las habilidades fundamentales de la asignatura aplica estrategias de precios y logra estrategias comunicacionales para empresas, productos o servicios.

Un resultado preciso es el producto integrador titulado Elaboración y distribución de platos típicos ecuatorianos en el centro histórico de Quito y otro denominado Eco Jeans. En ambos casos se evidenciaron las habilidades logradas por los estudiantes de diferentes carreras, lo que materializó el objetivo de la asignatura.

El resultado final fue que, con la puesta en marcha del plan de marketing en la empresa Eco Jeans se creó un emprendimiento innovador para la satisfacción del cliente. Mostró productos ecoamigables para el medio ambiente. Además, la empresa será redituable. Para este emprendimiento, los estudiantes de la carrera de "Gestión Empresarial" ocuparon los procesos de mercadeo; los de contabilidad, finanzas y auditoría por su parte, puntualizaron la 
gestión de las finanzas, mientras que los correspondientes al curso de diseño gráfico, elaboraron las propuestas de los pantalones, asumiendo la recuperación en nuevos modelos desde jeans ya usados. Este fue un resultado de la materialización del trabajo con el modelo de aprendizaje en grupos heterogéneos.

Otro resultado que develó la transformación percibida en los estudiantes fue el caso de la propuesta de un plan de marketing para el Mercado CotocoIlao, de la ciudad de Quito. El trabajo evidenció la habilidad de diseñar cuando partieron de un diagnóstico de la situación del mercado para apoyar la mejora en producto, precio, plaza, promoción y se presentó el plan ante los representantes del mercado.

Las acciones organizativas, los métodos y técnicas que emplearon los estudiantes develaron mejoría en el aprendizaje en los grupos heterogéneos B y B-1, lo que reafirmó la pertinencia. Su transferibilidad y posible extensión de este dependen de las experiencias de los docentes. El trabajo permitió encontrar una explicación teórica y una propuesta ante el fenómeno de los grupos heterogéneos en las universidades, fundamentalmente en aquellas que siguen la distribución de estudiantes de múltiples carreras para asumir las asignaturas básicas.

En conclusión, se confirmaron las ideas de Hernández (2013), Madariaga (2015) y García (2016) respecto al aprendizaje cooperativo y colaborativo. El empleo de una planificación de las actividades del grupo proporciona una eficiencia si esta se coordina de acuerdo con los niveles de aprendizaje. La propuesta de un "modelo de grupo de aprendizaje" puede constituir un referente para organizar el proceso de enseñanza-aprendizaje en grupos heterogéneos, como se constató en los resultados de dos grupos que recibieron la asignatura "Fundamentos del marketing" durante el periodo 39 en la sede Quito de la Universidad Metropolitana.

\section{REFERENCIAS BIBLIOGRÁFICAS}

Bondi, S. y Daher, T. (2016). Learning through personal connections: cogenerative dialogues in synchronous virtual spaces. Teaching in Higher Edu- cation. Disponible en https://doi.org/10.1080/135 62517.2016.1141288.

Feito, R. (2015). La enseñanza en grupos homogéneos y heterogéneos. Recuperado de https:// www.researchgate.net/publication/267832960_ la_ensenanza_en_grupos_homogeneos_y_heterogeneos.

Fernández de Haro, E. (2014). El trabajo en equipo mediante aprendizaje cooperativo. Departamento de Psicología Evolutiva y de la Educación. Recuperado de http://conexiones.dgire.unam. mx/wp-content/uploads/2017/09/El-trabajo-enequipo- mediante-aprendizaje-cooperativo-engrupos.pdf.

García, A. y Mena, J. (2016). Information Technology as a Way To Support Collaborative Learning: What In-Service Teachers Think, Know and Do. Journal of Information Technology Research, 9 (1), 1-17 https://doi.org/10.4018/JITR.2016010101. Recuperado de https://www.igi-global.com/article/information-technology-as-a-way-to-supportcollaborative-learning/149673?camid=4v1 .

Gómez-Pezuela, G. (2007). Desarrollo psicológico y aprendizaje. México: Ed. Trillas.

Hernández-Selles, N., González-Sanmamedy, M. y Muñoz-Carril, P.C. (2015). Teacher's roles in learning ecologies: looking into collaborative learning in virtual environments. Revista de Curriculum y Formación de Profesorado, 19, (2). Recuperado de https://recyt.fecyt.es/index.php/profesorado/ article/view/41523.

Jara, D.I., Ramírez, M.S. y Cabezas, M. (2016). Personal Social Network as a Facilitating Tool for Collaborative Knowledge Construction). ACM International Conference Proceeding Series. Recuperado de https://doi.org/10.1145/3012430.3012656.

Kagan, S. (1994). Cooperative learning. San Clemente: Resources for Teachers.

López, N. y González, P. (2014). Audioblogs and Tvblogs, Tools for Collaborative Learning in Journalism. Comunicar 21 (42), pp. 45-53. doi: 10.3916/c42-2014-04. 
Madariaga, E., Correa, F., Oria, J.M. y Walliser, J. (2015). Participation of students of maritime engineering in the naval exercise "MARSEC 2015" with website of the Spanish Navy. Interrnational Conference on Education \& Professional Development of Engineers in the Maritime Industry. Recuperado de www.rina.org.uk/hres/EPD15\%20 brochure\%20WEB.pdf.

Navarro, J.P., Sala, H. y Limbiati, V. (2013). Uso intensivo de herramientas de colaboración en línea en educación superior. Revista Electrónica de Didáctica en Educación Superior, 6. Recuperado de http://www.biomilenio.net/RDISUP/numeros/06/06\%20Navarro\%20Sala\%20et\%20al.pdf.

Peirats, J. y López, M. (2013). Los grupos interactivos como estrategia didáctica en la atención a la diversidad. En Ensayos, Revista de la Facultad de Educación de Albacete, 28. Recuperado de http:// www.revista.uclm.es/index.php/ensayos.

Piñeiro, T. y Costa, C. (2015). Alternate Reality Games (ARG). Contributions, Limitations, and Potentialities to the Service of the Teaching at the University Level. Revista Comunicar. Recuperado de https://doi.org/10.3916/C44-2015-15.

Pujolás, P. (2014). Agrupamiento heterogéneo del alumnado y atención a la diversidad: La estructura cooperativa de la actividad en el aula. Revista Perspectiva Escolar, 324.

\section{ANEXO 1}

\section{Instrumento de diagnóstico para la asignatura "Fundamentos del marketinga" en grupos hetero- géneos}

Estimado estudiante, para un mejor desempeño en la asignatura y lograr que adquiera conocimientos y habilidades se necesita su colaboración. En este sentido, le proponemos las siguientes interrogantes o situaciones en las que debe dar respuesta con toda libertad.

Nombre y apellidos

Edad

Carrera que estudia
Experiencia profesional actual

Los procesos de marketing son de vital importancia, ya que constituyen una manera de concebir y ejecutar las relaciones de intercambio que se establecen entre quienes generan bienes y servicios, y quienes los consumen. Fortalecen los niveles de satisfacción de ambos en la sociedad mediante el desarrollo, valoración, distribución y promoción por una de las partes de los bienes, servicios e ideas que la otra parte necesita.

1. Del anterior planteamiento seleccione y marque con una $\mathrm{X}$ las que considera válidas para su profesión:

- Asegura adecuada atención en su centro laboral al:

- Cliente

- Usuario

- Mercado de consumidores

- Los niveles de satisfacción en bienes o servicios que logra su centro laboral son:

- Altos

- Medios

- Bajos

- Identifica que en su centro laboral se tienen en cuenta los deseos y necesidades de:

- Clientes

- Usuarios

- Mercado de consumidores

2. De las siguientes definiciones y conceptos marque con una $X$ las que considere apropiadas y que correspondan con su profesión. 
El proceso de enseñanza-aprendizaje en grupos heterogéneos de la Universidad Metropolitana del Ecuador

Proceso social y administrativo mediante el cual los individuos y los grupos obtienen lo que necesitan y desean, creando e intercambiando valor con otros.

Proceso mediante el cual las empresas generan valor para los clientes y establecen relaciones sólidas con ellos, obteniendo a cambio valor de los clientes.

Herramienta que se encarga de los clientes, con la cual se busca una relación perdurable con ellos y al mismo tiempo atraer más clientes.

3. La oferta de marketing es cierta combinación de productos, servicios, información o experiencia que se ofrece a un mercado para satisfacer una necesidad o un deseo. El marketing consiste en acciones que se realizan para diseñar y mantener relaciones de intercambio deseables con audiencias meta, las cuales implican bienes, servicios, ideas u otros objetos.

Considera afirmativo lo anterior:

- Sí

- No

- Más o menos 